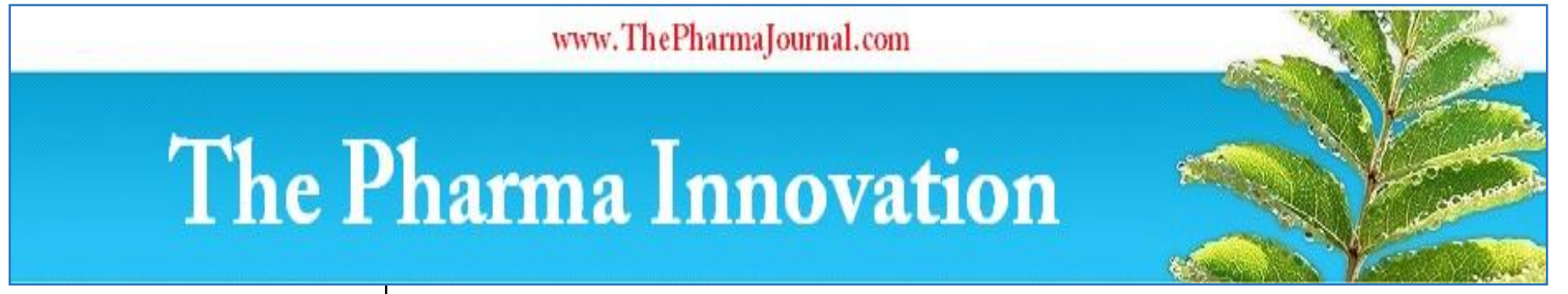

ISSN (E): 2277 - 7695

ISSN (P): 2349-8242

NAAS Rating: $\mathbf{5 . 0 3}$

TPI 2020; 9(10): 211-216

(C) 2020 TPI

www.thepharmajournal.com

Received: 16-08-2020

Accepted: 21-09-2020

\section{Ruchi Kumari}

PG, Department of Home

Science and Food and Nutrition,

Tilka Manjhi Bhagalpur

University, Bhagalpur, Bihar,

India

Mamta Kumari

PG, Department of Home

Science and Food and Nutrition,

Tilka Manjhi Bhagalpur

University, Bhagalpur, Bihar,

India
Corresponding Author:

Mamta Kumari

PG, Department of Home

Science and Food and Nutrition,

Tilka Manjhi Bhagalpur

University, Bhagalpur, Bihar,

India

\section{Factors affecting the choice of fast food among teenagers of Sabour block in Bhagalpur district of India}

\author{
Ruchi Kumari and Mamta Kumari
}

DOI: $\underline{\text { https://doi.org/10.22271/tpi.2020.v9.i10c.5230 }}$

\begin{abstract}
In recent days, there is rapid shift in consumer's life-style due to change in standard of living, work culture, hectic work schedule, and free-thinking and global perspective, as a consequence, fast foods impact our dietary pattern and routine food consumption. As fast food is generally considered rich in calorie, fat, sugar, salt and poor in other nutrients, it has contributed to rise of many non- communicable diseases, metabolic diseases such as obesity and over-weight, diabetes mellitus, hypertension and cardiovascular diseases among more common. The present study was conducted to find the factors affecting the choice of fast food (Indian Fast Food, IFF; Western Fast Food, WFF) among teenagers using questionnaire-cum-structured interview schedule. In response to the influence of nutritional information about fast foods on the teenager's choice of fast food, in case of IFF, the response was rarely (girls, 30\%, boys, 19.6\%), sometimes (girls, 40.4\%, boys, 45.6\%), most of time (girls, 20\%, boys, $20.8 \%$ ), and always (girls, $9.6 \%$, boys, $6.4 \%$ ), while in case of WFF, the response was rarely (girls, $20 \%$, boys, $19.2 \%$ ), sometimes (girls, $45.2 \%$, boys, $42.4 \%$ ), most of time (girls, 17.2\%, boys, 20.8\%), and always (girls, $15.2 \%$, boys, $6.4 \%$ ). In response to query on awareness for chemicals and preservatives present in fast food, respondents considered fast food as a healthy food in case of IFF response was yes (girls, 61.2\%, boys, 53.6\%), and no (girls, 37.2\%, boys, $49.2 \%$ ); while in case of WFF Always (girls, $57.6 \%$, boys, $46.4 \%$ ) and no (girls, $37.2 \%$, boys, $49.2 \%$ ). Regarding having prior knowledge of nutritive value of fast foods, in case of IFF, the response was as follows, "Yes, it has high nutritive value" (girls, $40.4 \%$, boys, $44.4 \%$ ), and "Yes, it has low nutritive value" (girls, $22 \%$, boys, $19.6 \%$ ), "no I don't know" (girls, 35.2\%, boys, 26.8\%); while in case of WFF, "Yes it is high in calorie" (girls, 17.6\%, boys, 17.2\%), "Yes it has low calorie" (girls, 35.6\%, boys, 31.2\%), and " no I don't know" (girls, 43.2\%, boys, 45.6\%). This valuable information would be useful for preparing strategies related to fast food consumption among teenagers and their social, health and economical issues.
\end{abstract}

Keywords: Fast food, nutritional information, teenagers

\section{Introduction}

The rise of fast food industry has changed the global urban food culture in India to some extent. In India fast food culture emerged post-independence, however, over a period of time with a growth in the number of nuclear families as well working parents, increasing per capita income as well as globalization. The fast food culture gained prominence with the liberalized Indian economy post 1990's, several international brands has made their presence in Indian fast food market with their outlets in cities largely functioning in shopping malls and other public areas. India's fast-food industry is expanding at the rate of $40 \%$ every year. India ranks $10^{\text {th }}$ in the fast food per capita spending, and it is $2.1 \%$ of expenditure in annual total spending (Ashakiran et al., 2012) ${ }^{[1]}$. In recent times, the fast food industry is adapted to Indian food requirement and is growing very fast. As fast food is generally considered rich in calorie, fat, sugar, salt and poor in other nutrients, it has contributed to rise of many non-communicable diseases, metabolic diseases such as obesity and over-weight, diabetes mellitus, hypertension and cardiovascular diseases among more common. Banerjee et al. (2013) ${ }^{[2]}$ stated that fast food is by-product of technological advances in food processing, newly invented food additives and the techniques for preservation. Though fast food saves time, it doesn't save us from consuming fats, sodium and preservatives and as a consequence a bad health for ourselves. India also has long tradition foods with variety of recipes that still prevail in various part of country. Out of various foods consumed across the world, "fast food" is one that is becoming popular among masses, especially young generations, thereby it is one of the fastest 
Growing industries that impact teenager's health. Therefore, the present study was conducted to know the factors affecting the choice of fast food and help preparing strategies related to advantages and disadvantages of fast food consumption among teenagers.

\section{Methods}

All the methods used in the present study are given in this chapter. The statistical method was used to get data analyzed and the numerical data prepared to draw the conclusion. Statistical methods simplify unwisely masses of complex data by process of tabulation, summarization, and possibilities from the nature of the data available. It comprised all aspect of data including data collection in terms of the design of surveys and experiments.

\subsection{Data collection}

The present study was conducted at Sabour Block of Bhagalpur district (Bihar). A convenient selection of ten schools/colleges was made for study purpose. Total 500 respondents, which include 250 boys and 250 girls $(\sim 50$ students from each school/college) of age 12-18 years, were randomly selected for present study. The permission to conduct study was obtained from the respective school principals. Also, the consent from the parents or guardians in addition to assent from each student was obtained after providing them information and purpose of this study. The questionnaires-cum-structured interviews were conducted to as many consenting students chosen randomly in each class. The questionnaire-cum structured interview schedule prepared in English and Hindi was pretested to students before its usage in the study. It contained structured questions on fast food consumption. The list of common food stuffs labeled as fast food was also detailed in the questionnaire for the easy understanding of the participants. Other questions such as type of fast food consumed, source of information about fast foods, reasons for consumption, awareness about its nutritional benefits and health hazards, size and frequency of fast foods and socio-economic status of family, etc. were mainly enquired. Grossly incompletely filled forms were excluded from analysis.

\subsection{Pilot survey}

The main objectives of the pilot survey were as follows: firstly, to check that the questions we have asked are providing us the relevant information regarding the main objectives of our survey or not. Secondly, to check the various factors like approximate time needed to fill up a schedule, for getting the feedback of the respondent if they find any question irrelevant or illogical to check what type of response we are getting while doing the survey i.e. the respondent should not find the question uninteresting. Also the pilot survey helped us in finding out the most effective way to ask questions so as to get the best possible response from the respondent. Moreover, the pilot survey helped in pre-testing the schedule and to get an idea about the procedure of the survey. Ten schedules were given to respondents (teenagers) prior to actual survey.

\subsection{Tools of data collection and analysis:}

Data was collected with the help of questionnaire-cumstructured interview schedule. Accordingly, wherever necessary information from secondary sources such as books, journals, newspapers, and internet was used. Collected data from the field was properly analyzed and presented in the tables and graphic forms with the help of several statistics tools as per the need of study. On the basis of collected data to draw different conclusions and inference, the statistical procedure were adopted, (1) Tabulation of data by using frequency tables and cross tabulation, (2) Graphical representation, and (3) Testing of Hypotheses by applying the Chi-square $\left(\chi^{2}\right)$ test.

\subsection{Statistical Analysis}

A sample of 500 students (including both boys and girls) from different schools and colleges residing in urban areas were randomly selected for the present study. The information gathered and obtained for the study was carefully construed and condensed into a master chart. The data was then arranged, tabulated and discussed upon and was presented with the help of tables and figures. The data was further statistically analyzed. Chi-Square $\left(\chi^{2)}\right.$ Test of independence was followed as it is a procedure for testing if two categorical variables are related in some population. The P-value was calculated using P-value Calculator available online (https://www.socscistatistics.com/pvalues/chidistribution.aspx ;https://www.graphpad.com/quickcalcs/pvalue1.cfm).

\section{Results and Discussion}

Consumption of diet high in sugar, saturated fat, salt and calorie content in children can lead to early development of obesity, hypertension, dyslipidemia and impaired glucose tolerance. The concerns with fast food consumption in developing countries also include poor hygiene during preparation storage and handling leading to microbiological contamination. Fast foods have high level of fat and sugars that are not only unhealthy but addictive and that creates a vicious cycle making it hard for children to choose healthy

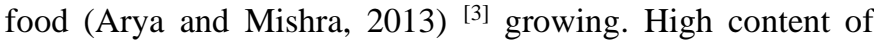
trans-fat in commercially available fast foods predispose children to risk of future heart diseases (Asgary et al., 2009) [4]. Energy density of fast food is more than twice the recommended daily allowance for children (Printice and Jebb, 2003) [5]. Fast food intake leads to higher proportion of calories being derived from total and saturated fat. Moreover, the micronutrient content (carotene, vitamin A, vitamin C) of the fast food is also low. Low levels of calcium and magnesium is also found in the fast foods. Therefore experts attributed the current childhood obesity epidemic to fast foods (Ashakiran and Deepthi, 2012) ${ }^{[1]}$. The association between body weight and health has received considerable attention and has major potential public health implications. BMI $\left(\mathrm{Kg} / \mathrm{m}^{2}\right)$ is a measure of body fat based on height and weight that applies to adult men and women. The fast foods affects BMI reveals an estimate of body fat and considered to be gauge of risk for diseases that can occur with more body fat.

\subsection{Influence of nutritional information on choice of fast food to the respondents}

Regarding query on influence of nutritional information on choice of fast food in case of IFF, the response was rarely (girls, 30\%, boys, 19.6\%), sometimes (girls, 40.4\%, boys, $45.6 \%$ ), most of time (girls, 20\%, boys, 20.8\%), and always (girls, 9.6\%, boys, 6.4\%) (Table 1; Fig. 1A).; While in case of WFF, the response was rarely (girls, 20\%, boys, 19.2\%), sometimes (girls, $45.2 \%$, boys, $42.4 \%$ ), most of time (girls, $17.2 \%$, boys, $20.8 \%$ ), and always (girls, $15.2 \%$, boys, $6.4 \%$ ) (Table 1; Fig. 1B). The highest response for rarely/sometimes 
in case of both foods shows that teenagers need to be educated about the importance of getting informed about nutritional content of fast foods they eat and their relatedhealth issues.

Table 1: Influence of nutritional information on choice of fast food to the respondents

\begin{tabular}{|c|c|c|c|c|c|c|}
\hline Variables & \multicolumn{4}{|c|}{ Values ${ }^{\#}$} & \multirow{2}{*}{ Chi-square } & \multirow{2}{*}{$\mathbf{P}$-value } \\
\hline & \multicolumn{3}{|c|}{ Girls $(n=250)$} & Boys $(n=250)$ & & \\
\hline Rarely & 30 & 20 & 19.6 & 19.2 & \multirow[t]{3}{*}{ Girls, $\chi 2=9.28$} & \multirow{5}{*}{$\begin{array}{c}\text { P-value } 0.025679 . \\
\text { The result is significant. } \\
p<0.05,3 \mathrm{df} \text { P-value } \\
0.660456 \text {. The result is not significant, } \\
p<0.05,3 \mathrm{df}\end{array}$} \\
\hline Sometimes & 40.4 & 45.2 & 45.6 & 42.4 & & \\
\hline Most of the time & 20 & 17.2 & 20.8 & 20.8 & & \\
\hline Always & 9.6 & 15.2 & 6.4 & 6.4 & \multirow[t]{2}{*}{ Boys, $\chi 2=1.59$} & \\
\hline & & & & & & \\
\hline
\end{tabular}

\#Data are presented as No. (\%)

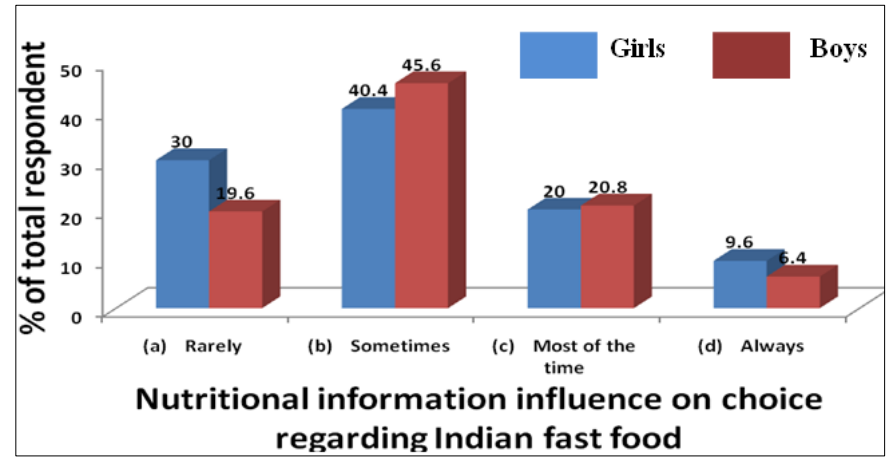

A

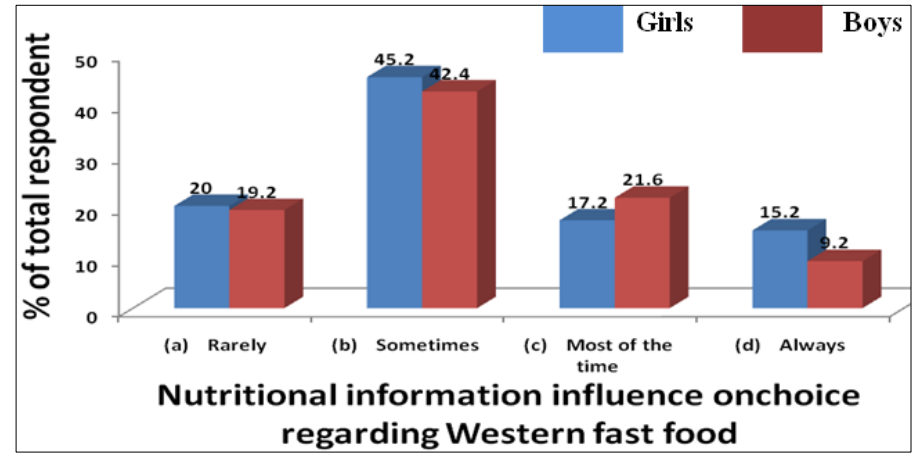

B

Fig 1: Graphical representation of influence of nutritional information on choice of IFF (A) and WFF (B) to the respondents

In case of both the foods (IFF and WFF), regarding query on influence of nutritional information on choice of fast food, the highest response was rarely/sometimes. It shows that teenagers need to be educated about the nutritional values of fast foods and their impacts on health.

\subsection{Awareness for chemicals and preservatives present in fast food}

In a query on presence of chemicals and preservatives in fast food and whether it is a healthy food in case of IFF response was yes (girls, $61.2 \%$, boys, 53.6\%), and no (girls, $37.2 \%$, boys, $49.2 \%$ ) (Table 2; Fig. 2A); while in case of WFF the response was yes (girls, $57.6 \%$, boys, 46.4\%) and no (girls, $37.2 \%$, boys, $49.2 \%$ ) (Table 2; Fig. 2B). This observation clearly shows the need of awareness among teenagers, as large percentages of teenagers are away from the facts about the ill-effects of fast foods on health.

Table 2: Awareness for chemicals and preservatives present in fast food to the respondents

\begin{tabular}{|c|c|c|c|c|c|c|}
\hline Variables & \multicolumn{4}{|c|}{ Values $^{\#}$} & \multirow{2}{*}{ Chi-square } & \multirow{2}{*}{$\mathbf{P}$-value } \\
\hline & & $\mathbf{s}(\mathbf{n}=1$ & & Boys $(n=250)$ & & \\
\hline Yes & 61.2 & 57.6 & 53.6 & 46.4 & & P-value 0.74591 \\
\hline No & 37.2 & 37.2 & 42 & 49.2 & $\begin{array}{l}\text { Girls, } \chi 2=0.10 \\
\text { Boys, } \chi 2=2.71\end{array}$ & $\begin{array}{c}\text { The result is not significant. } \\
\quad p<0.05,1 \mathrm{df} \\
\text { P-value } \\
0.099284, \\
\text { The result is not significant, } \\
p<0.05,1 \mathrm{df}\end{array}$ \\
\hline
\end{tabular}

${ }^{\#}$ Data are presented as No. (\%)

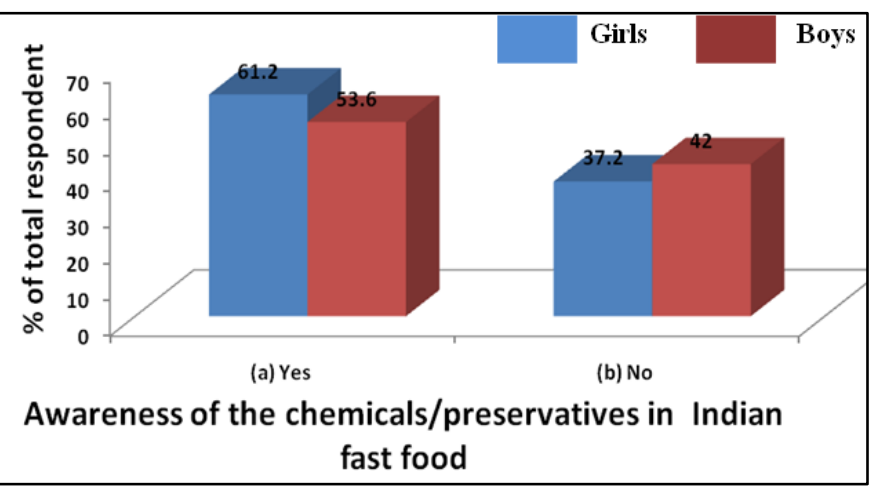

A

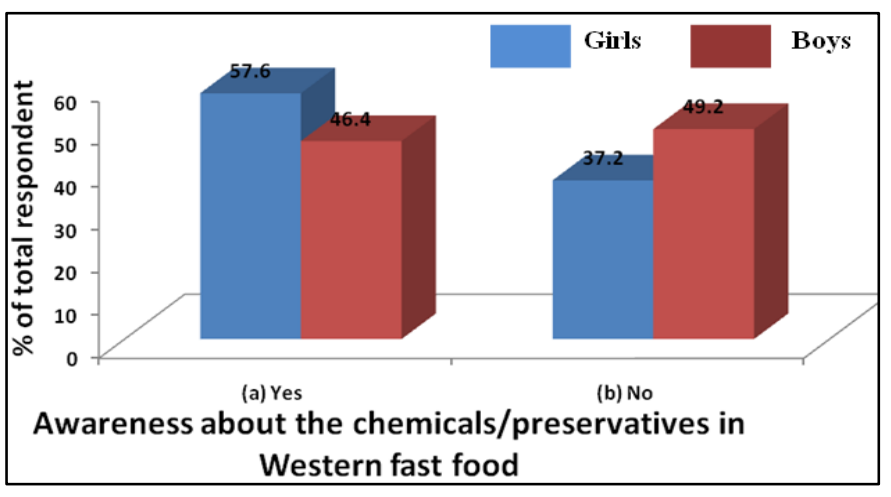

B

Fig 2: Graphical representation of awareness for chemicals and preservatives present in IFF (A) and WFF (B) to the respondents 


\subsection{Knowledge of nutritive value of fast foods}

Regarding having prior knowledge of nutritive value of fast foods, in case of Indian fast food, the response was as follows, "Yes, it has high nutritive value" (girls, 40.4\%, boys, 44.4\%), and "Yes, it has low nutritive value" (girls, 22\%, boys, 19.6\%), "no I don't know" (girls, 35.2\%, boys, 26.8\%) (Table
3; Fig. 3A); while in case of WFF, "Yes it is high in calorie" (girls, 17.6\%, boys, 17.2\%), "Yes it has low calorie" (girls, $35.6 \%$, boys, $31.2 \%$ ), and " no I don't know" (girls, $43.2 \%$, boys, 45.6\%) (Table 3; Fig. 3B). These responses are reflection of lack of information/knowledge about the nutritive value of fast foods among teenagers.

Table 3: Knowledge of the nutritive value of IFF and WFF by respondents

\begin{tabular}{|c|c|c|c|c|c|c|}
\hline \multirow[t]{2}{*}{ Variables } & \multicolumn{4}{|c|}{ Values ${ }^{\#}$} & \multirow{2}{*}{ Chi-square } & \multirow{2}{*}{$\mathbf{P}$-value } \\
\hline & & \multirow{2}{*}{$\begin{array}{c}\text { Girls }(n=250) \\
\text { IFF WFF }\end{array}$} & \multicolumn{2}{|c|}{ Boys $(n=250)$} & & \\
\hline & & & & FF & & \\
\hline Yes, it has high nutritive value & 40.4 & 17.6 & 44.4 & 17.2 & & P-value \\
\hline Yes, it has low nutritive value & 22 & 35.6 & 19.6 & 31.2 & Girls $\gamma 2=3245$ & $<0.00001$ \\
\hline No, I don't know & 35.2 & 43.2 & 26.8 & 45.6 & Boys, $\chi 2=48.72$ & $\begin{array}{c}p<0.05,2 \mathrm{df} \\
\mathrm{P} \text {-value } \\
<0.00001 . \\
\text { The result is significant, } \\
p<0.05,2 \mathrm{df}\end{array}$ \\
\hline
\end{tabular}

${ }^{\#}$ Data are presented as No. (\%)

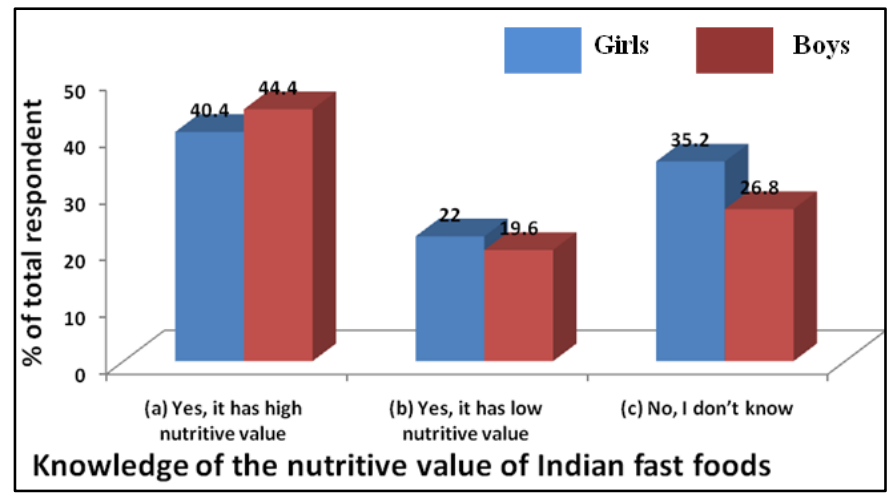

A

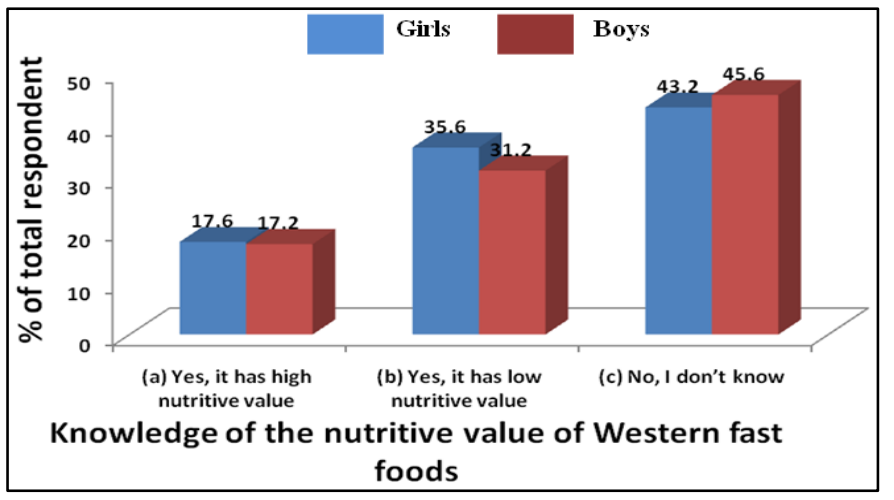

B

Fig 3: Graphical representation of knowledge of the nutritive value of IFF (A) and WFF (B) by respondents

\subsection{Reason for attraction to fast food}

The attraction for IFF was due to convenience (girls, $31.6 \%$ and boys, 20.8\%) followed by advertisement (girls, $18.8 \%$ and boys, $14.4 \%$ ), changing life style/Global culture (girls, $9.2 \%$ and boys, $17.6 \%$ ), reputation among friends (girls, $5.6 \%$ and boys, $14.4 \%$ ), and lesser due to relaxation (girls, $8.8 \%$ and boys, $7.6 \%$ ), and promotional offers (girls, $0.8 \%$ and boys, $2.8 \%$ ) (Table 4; Fig. 4A). The attraction for WFF was due to convenience (girls, 22.8\% and boys, 6.8\%), advertisement (girls, $23.6 \%$ and boys, $27.2 \%$ ), changing life style/global culture (girls, $10.4 \%$ and boys, $17.2 \%$ ), reputation among friends (girls, 2.4\% and boys, 19.2\%), lesser due to relaxation (girls, $2.8 \%$ and boys, $7.6 \%$ ), promotional offers (girls, 3.2\%; boys, 5.6 (Table 4; Fig. 4B). Hence, relaxation and promotional offers are not so enticing as far as fast food concern in both the gender.

Table 4: Factor that attracted for IFF and WFF to respondents

\begin{tabular}{|c|c|c|c|c|c|c|}
\hline \multirow{3}{*}{ Variables } & \multicolumn{4}{|c|}{\begin{tabular}{|c|} 
Values $^{\#}$ \\
\end{tabular}} & \multirow{3}{*}{ Chi-square } & \multirow{3}{*}{ P-value } \\
\hline & \multicolumn{2}{|c|}{\begin{tabular}{|c|} 
Girls $(n=250)$ \\
\end{tabular}} & \multicolumn{2}{|c|}{ Boys $(n=250)$} & & \\
\hline & IFF & WFF & IFF & WFF & & \\
\hline Advertisement & 18.8 & 23.6 & 14.4 & 27.2 & \multirow{7}{*}{$\begin{array}{l}\text { Girls, } \chi 2=84.51 \\
\text { Boys, } \chi 2= \\
33.55\end{array}$} & \multirow{7}{*}{$\begin{array}{l}\text { The P-value is }<.00001 \\
\text { The result is significant at } p<0.05,6 \mathrm{df} \\
\text { The } \mathrm{P} \text {-value is }<.00001 \text {. The result is significant at } p<0.05,6 \mathrm{df}\end{array}$} \\
\hline Promotional offers & 0.8 & 3.2 & 2.8 & 5.6 & & \\
\hline Convenience & 31.6 & 22.8 & 20.8 & 6.8 & & \\
\hline Relaxation & 8.8 & 2.8 & 7.6 & 7.6 & & \\
\hline Changing life style/Global culture & 9.2 & 10.4 & 17.6 & 17.2 & & \\
\hline Reputation among friends & 5.6 & 2.4 & 14.4 & 19.2 & & \\
\hline Any other & 24 & 32.4 & 20.4 & 15.2 & & \\
\hline
\end{tabular}

"Data are presented as No. (\%) 


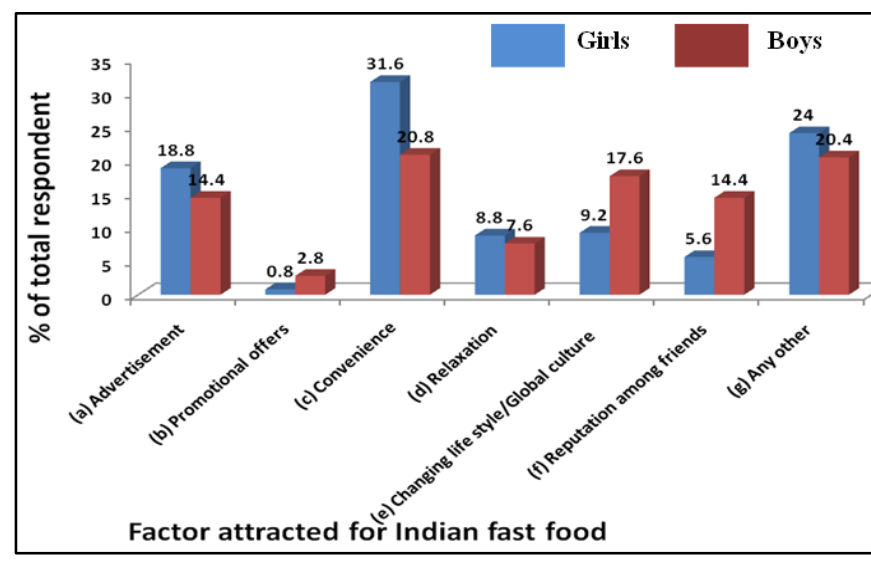

A

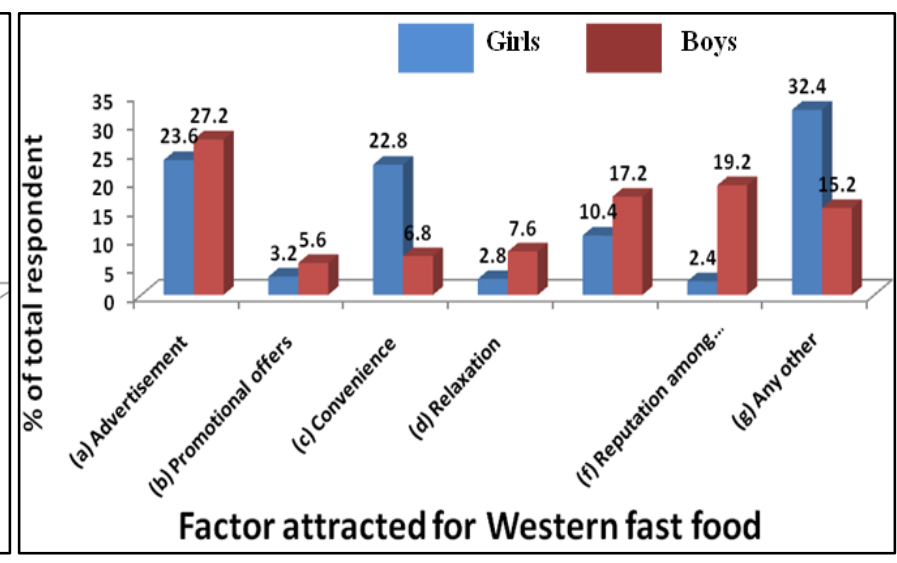

B

Fig 4: Graphical representation of factor that attracted for IFF (A) and WFF (B) to respondents

\subsection{Branding effect on choosing fast food}

Branding affect on choosing IFF, respondents "Agreed" (girls, 64\% and boys, 55.2\%), neutral (girls, 22\% and boys, $18.4 \%$ ), and disagreed (girls, $13.2 \%$ and boys, 22\%) (Table 5; Fig. 5A). Branding affect on choosing WFF, respondents "Agreed" (girls, 51.6\%; boys, 55.2\%), neutral (girls, 28.4\%; boys, 21.6\%), and disagreed (girls, 17.6\%; boys, 15.6\%) (Table 5; Fig. 5B). Branding affected the choice of having fast food, in case of IFF responded "Agreed" comprised girls
(64\%) and boys (55.2\%), and in case of WFF (girls, 51.6\% and boys, 55.2\%), (Table 5; Fig. 5A; Fig. 5B). Prior survey reports showed that heavier $\mathrm{TV}$ use and more frequent commercial TV viewing were independently associated with more positive attitudes toward junk food and higher junk food consumption (Dixon et al., 2007). Furthermore, this survey also found that ads for nutritious foods promote selected positive attitudes and beliefs related to such foods.

Table 5: Branding affecting the choice of IFF and WFF on respondents

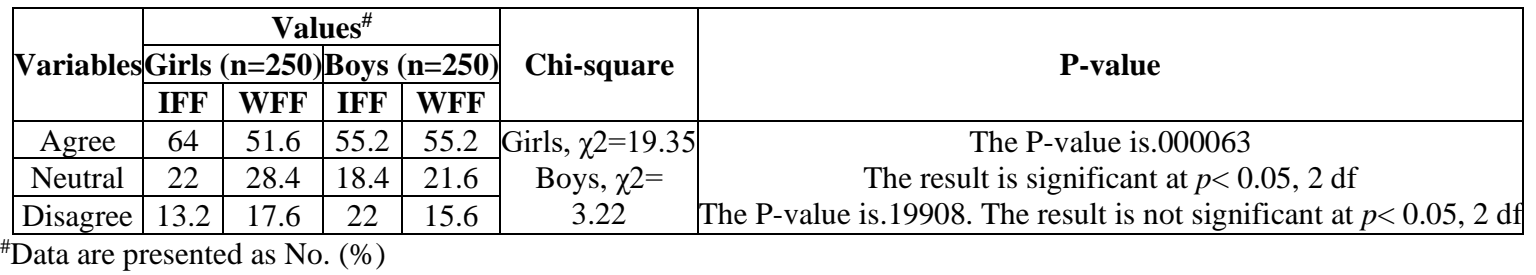

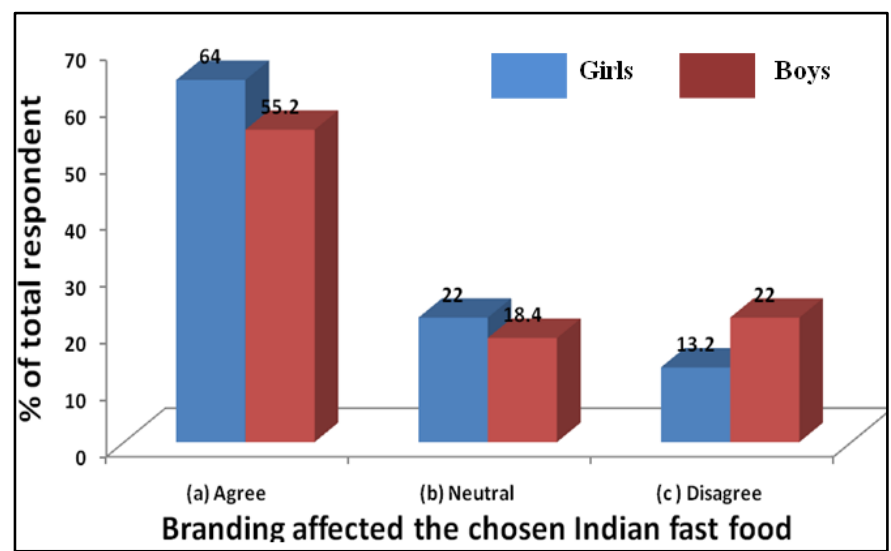

A

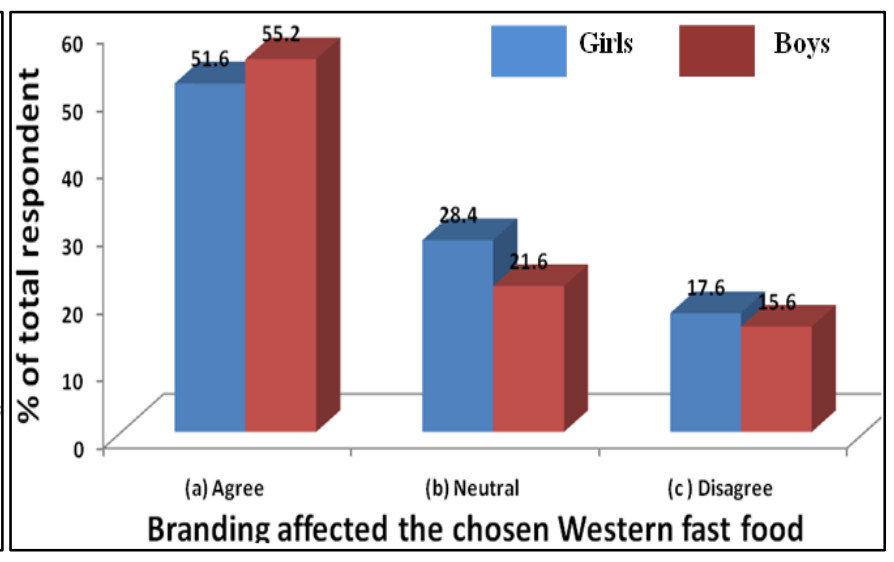

B

Fig 5: Graphical representation of branding affecting the choice of IFF (A) and WFF (B) on respondents

Earlier report suggested that the proximity of fast food joints to households could also predispose to increased consumption (Kaushik et al., 2011) ${ }^{[7]}$. Several studies emphasized on the importance of establishing a healthy eating behavior among children and adolescents, and interventions such as improving the convenience of healthy fast foods in school canteens and neighborhood stores, establishing policies to increase the cost of unhealthy fast food; need of stronger policies to provide healthier meals to students at schools by limiting access to low-nutrient, energy-dense foods and increasing the frequency, intensity, and duration of physical activity (Story et al., 2009; Kaushik et al., 2011) ${ }^{[7,8]}$.

\section{Conclusion}

In case of both the foods (IFF and WFF) regarding query on influence of nutritional information on choice of fast food, the highest response was rarely/sometimes. Likewise, in response to a query to find the awareness level for chemicals and preservatives present in fast food and whether it is a healthy food, the response was highest for fast food as healthy food, it shows that teenagers needs to be educated about the nutritional value and ill-effects of these chemicals and 
preservatives in fast foods. Also, regarding having prior knowledge of nutritive value of fast foods, in case of both the fast food, the response shows lack of information among teenagers. While in response to the reason for attraction to fast food among teenagers was highest due to convenience followed by advertisement and changing life style/global culture, and the factors like relaxation and promotional offers are not so enticing as far as fast food concern in both the gender. Further, it was also found that the branding of fast foods do affect the choice of teenagers. Hence, taken together, the present study would be helpful in planning strategies related to increasing trend of fast food consumption among teenagers, and their possible social, health and economical issues.

\section{Acknowledgement}

Authors thank the PG Department of Home Science-Food and Nutrition, Tilka Manjhi Bhagalpur University, Bhagalpur for their all support during the study.

\section{References}

1. Ashakiran, Deepthi R. Fast foods and their impact on health. Journal of Krishna Institute of Medical Sciences University. 2012; 1:7-15.

2. Banerjee S, Joglekar A, Kundle S. Consumer awareness about convenience food among working and nonworking women. Int. J Sci Res. 2013; 2(10):1-4.

3. Arya G, Mishra S. Effect of junk food \& beverages on Adolescent's health. IOSR J Nur. Health Sci. 2013; 1:2632.

4. Asgary S, Nazari B, Sarrafzadegan N, Parkhideh S, Saberi S, Esmaillzadeh A et al. Evaluation of fatty acid content of some Iranian fast foods with emphasis on trans fatty acids. Asia Pac. J. Clin. Nutr. 2009; 18:187-92.

5. Printice AM, Jebb SA. Fast foods, energy density and obesity: a possible mechanistic link. Obesity Rev. 2003; 4:187-94.

6. Dixon HG, Scully ML, Wakefield MA, White VM, Crawford DA. The effects of television advertisements for junk food versus nutritious food on children's food attitudes and preferences. Soc. Sci. Med. 2007; 65:131123.

7. Kaushik JS, Narang M, Parakh A. Fast Food Consumption in Children. Indian Pediatrics. 2011; 97(48):97-101.

8. Story M, Nanney MS, Schwartz MB. Schools and obesity prevention: creating school environments and policies to promote healthy eating and physical activity. Milbank Q. 2009; 87(1):71-100. 